\title{
Fractional telegrapher's equation from fractional persistent random walks
}

\author{
Jaume Masoliver* $^{*}$ \\ Departament de Física Fonamental, Universitat de Barcelona, Diagonal, 647, E-08028 Barcelona, Spain
}

(Received 16 March 2016; published 3 May 2016)

\begin{abstract}
We generalize the telegrapher's equation to allow for anomalous transport. We derive the space-time fractional telegrapher's equation using the formalism of the persistent random walk in continuous time. We also obtain the characteristic function of the space-time fractional process and study some particular cases and asymptotic approximations. Similarly to the ordinary telegrapher's equation, the time-fractional equation also presents distinct behaviors for different time scales. Specifically, transitions between different subdiffusive regimes or from superdiffusion to subdiffusion are shown by the fractional equation as time progresses.
\end{abstract}

DOI: 10.1103/PhysRevE.93.052107

\section{INTRODUCTION AND GENERAL SCOPE}

Transport of particles through a continuous medium is described by a transport equation which in its most general form can be a nonlinear integro-differential equation [1] with, in many practical cases, an imperfectly known scattering kernel [2]. The difficulty of numerical solutions to reproduce relevant qualitative characteristics of the transport process, as well as their use in applications, has traditionally led to the search for simpler approximations which are analytically tractable. Perhaps the most used of such approximations consists in modeling transport by diffusion processes. In such a case the transport equation reduces (in the force-free case) to the diffusion equation,

$$
\frac{\partial p}{\partial t}=D \nabla^{2} p
$$

where $p(\mathbf{r}, t)$ is the probability density function of the diffusing particle to be at $\mathbf{r}$ at time $t$ and $D$ is the diffusion coefficient. A salient characteristic of any diffusion process is that the mean square deviation grows linearly with time,

$$
\left\langle|\Delta \mathbf{r}(t)|^{2}\right\rangle=D t,
$$

where $\Delta \mathbf{r}(t)=\mathbf{r}(t)-\langle\mathbf{r}(t)\rangle$. Another major characteristic is the Gaussian nature of the process. Indeed, assuming the particle is initially at the origin, $p(\mathbf{r}, 0)=\delta(\mathbf{r})$, the solution to Eq, (1) is

$$
p(\mathbf{r}, t)=\frac{1}{(4 \pi D t)^{3 / 2}} e^{-r^{2} / 4 D t} .
$$

Despite its simplicity, the diffusion equation has some shortcomings in practical situations, one of them being its inability to account for ballistic motion as well as being inaccurate near boundaries. All of this implies limitations near the interfaces and in the description of early-time effects when the flux of ballistic particles may not be negligible [2].

One step forward in furnishing a better approximation to the full transport equation is provided by the telegrapher's equation (TE). Let us first remark that in the diffusive approximation the propagator of the particle instantaneously spreads out from $\delta(\mathbf{r})$ to the Gaussian form given above. That is to say, there is a nonzero probability for the diffusing particle

*jaume.masoliver@ub.edu to be found outside the spherical region $|\mathbf{r}|>c t$, where $c$ is the speed of light in the vacuum. In other words, the ordinary diffusion equation is not compatible with special relativity [3]. Although there is no unambiguous and widely accepted relativistic generalization of the diffusion equation, it is clearly recognized that the TE is a step forward in the correct direction [4-6].

In its isotropic form and in the absence of an external field, the TE reads $[2,7]$

$$
\frac{\partial^{2} p}{\partial t^{2}}+\frac{1}{T} \frac{\partial p}{\partial t}=c^{2} \nabla^{2} p,
$$

where $T>0$ is a characteristic time and $c>0$ is a characteristic speed. This is a hyperbolic equation which as $T \rightarrow \infty$ with $c$ fixed becomes the wave equation and as $T \rightarrow 0$ and $c \rightarrow \infty$ with $c^{2} T \rightarrow D$ finite, it turns into the diffusion equation. Equation (2) thus possesses wave and diffusion features, and it describes "diffusion with finite propagation velocity" [4,7]. This duality becomes even more manifest not only in the time behavior of the TE itself but also in the mean square deviation of the diffusing particle. One can easily see by scaling time with $T$ that, initially as $t \rightarrow 0$ (i.e., $t \ll T$ ), the TE approaches the wave equation while asymptotically as $t \rightarrow \infty(t \gg T)$ moves toward the diffusion equation. This leads to [2,7]

$$
\left\langle|\Delta \mathbf{r}(t)|^{2}\right\rangle \sim t^{2}(t \rightarrow 0), \quad\left\langle|\Delta \mathbf{r}(t)|^{2}\right\rangle \sim t(t \rightarrow \infty),
$$

showing the transition from ballistic motion to diffusive motion as time progresses [2].

The TE first appeared in the nineteen century within the context of electrodynamics with the works of Kelvin and Heaviside [2,7]. It is very useful in thermodynamics [8-11], population dynamics [12], and random walk theory where the $\mathrm{TE}$ is the master equation for the one-dimensional persistent random walk [13]. It also describes the probability density of one-dimensional random processes driven by the random telegraph signal $[14,15]$. In the context of transport theory, the three-dimensional TE is the so-called $\mathrm{P}_{1}$ approximation to the full transport equation for which the basic assumption is that the change in the direction of motion due to a single scattering is small $[1,2,16,17]$.

For more than two decades so-called "anomalous transport" has been the object of intense research in many branches of physics (for complete reports on the subject, see Refs. [18-25] and references therein, and see also a less technical but excellent introduction in Ref. [26]). The concept first appeared 
in the theory of random processes and specifically within continuous time random walks, a powerful technique developed by Montroll and Weiss 50 years ago [27], which was first applied to anomalous diffusion of charge carriers by Scher and Montroll in the 1970s [28,29].

Anomalous diffusion appears in transport through extremely disordered systems such as random media and fractal structures [30] and its most distinctive characteristic is that the mean square deviation follows the asymptotic law $[19,20]$ :

$$
\left\langle|\Delta \mathbf{r}(t)|^{2}\right\rangle \sim t^{\alpha}
$$

$(t \rightarrow \infty)$, where $\alpha>0$ is any positive real number. When $0<$ $\alpha<1$ the transport regime is termed as subdiffusive, and $\alpha=$ 1 corresponds to the (normal) diffusive regime while $\alpha>1$ is superdiffusive. Within the diffusive approximation and in the force-free case, the anomalous transport process is described by a fractional diffusion equation (FDE), which can be written as

$$
\frac{\partial^{\alpha} p}{\partial t^{\alpha}}=D \nabla^{2 \gamma} p
$$

$(0<\alpha \leqslant 1,0<\gamma \leqslant 1)$, where $\partial^{\alpha} / \partial t^{\alpha}$ is the fractional Caputo derivative and $\nabla^{2 \gamma}$ is the Riesz-Feller fractional Laplacian [31-35] (see Sec. IV for a definition of these operators).

In the case of particles diffusing under the influence of an external field of force, the FDE (4) is replaced by a fractional Fokker-Plank equation. We will not treat this case here, and we refer the interested reader to the literature for further information [20,22].

The mathematical properties of the solutions to the FDE (4) have been exhaustively studied and very clearly exposed by Mainardi and collaborators [33-35]. One of these properties is the scaling relation $[19,34,36,37]$

$$
p(\mathbf{r}, t)=t^{-\alpha / 2 \gamma} f\left(\frac{\mathbf{r}}{t^{\alpha / 2 \gamma}}\right),
$$

which results in the following scaling of the mean square displacement (assuming a symmetrical process, i.e., $\langle\mathbf{r}(t)\rangle=0$ ) [36]:

$$
\left\langle r^{2}(t)\right\rangle=M t^{\alpha / \gamma},
$$

showing that subdiffusion appears when $\alpha<\gamma$ and superdiffusion when $\alpha>\gamma$. Although very appealing, Eq. (6) has a limited range because $M$ turns out to be infinite when $\gamma \neq 1$ and the mean square displacement loses its sense except when $\gamma=1[36]$.

When $\gamma=1$ but $\alpha$ is not an integer we have the so-called "time-fractional diffusion," the case $0<\alpha<1$ corresponding to subdiffusion while $\alpha>1$ to superdiffusion. When $\alpha=1$ but $\gamma$ is not integer, the FDE (4) describes a Levy process; this case is always associated to superdiffusion, and it is termed "space-fractional diffusion" [22,36].

Let us recall that the formulation of fractional transport was first addressed within the continuous time random walk (CTRW) formalism [28,29]. In consequence derivations of the FDE are chiefly based on that formalism, although alternative approaches are based on master equations or (fractional) Chapman-Kolmogorov expansions [20].

In CTRW models a particle staying at $\mathbf{r}_{0}$ makes initially a random jump to $\mathbf{r}_{0}+\mathbf{r}$, then waits there during a random time interval $t$ and randomly jumps to a new position, waits there another (random) interval of time and makes another (random) jump, and so on. The walk is determined by two probability density functions, $\psi(t)$ for waiting time intervals and $g(\mathbf{r})$ for position jumps. Waiting times and jumps are generally assumed to be independent of each other [27] (though this is not the case of processes such as Lévy walks where jumps and waiting time are correlated [20,22]). The probability density function (PDF) of the diffusing particle, $p(\mathbf{r}, t)$, is related to $\psi(t)$ and $g(\mathbf{r})$ by means of the celebrated Montroll-Weiss equation for the joint Fourier-Laplace transform $\hat{\tilde{p}}(\boldsymbol{\omega}, s)$ of the PDF of the particle in terms of the Laplace transform, $\hat{\psi}(s)$, of the waiting-time density, and the Fourier transform, $\tilde{g}(\boldsymbol{\omega})$, of the jump density. Assuming that initially $p(\mathbf{r}, 0)=\delta(\mathbf{r})$, the Montroll-Weiss equation reads [15,27]

$$
\hat{\tilde{p}}(\omega, s)=\frac{[1-\hat{\psi}(s)] / s}{1-\hat{\psi}(s) \tilde{g}(\omega)} .
$$

The FDE (4) is obtained from Eq. (7) in the "fluid limit" [36] (i.e., large times and distances [38]) after assuming [20,35,36]

$$
\begin{gathered}
\hat{\psi}(s)=1-(T s)^{\alpha} \cdots \quad(s \rightarrow 0), \\
\tilde{g}(\omega)=1-(L|\omega|)^{2 \gamma} \cdots \quad(|\omega| \rightarrow 0),
\end{gathered}
$$

where $0<\alpha \leqslant 1,0<\gamma \leqslant 1, T$ and $L$ are positive constant parameters measured in units of time and length, respectively, and $D=L^{2 \gamma} / T^{\alpha}$.

Like the ordinary diffusion equation (1), the FDE (4) does not account for possible different dynamics of the diffusing particle at short and long times, which, as we have mentioned, appears in some experimental settings [39]. As seen above, the TE does possess this characteristic, which implies a transition from ballistic motion at short times to diffusion at long times.

The main objective of this paper is to generalize the TE to allow for anomalous transport. Following the footsteps of the FDE we will derive the space-time fractional telegrapher's equation (FTE) in one dimension from the continuous-time persistent random walk. We also obtain the characteristic function of the space-time fractional process and study some particular cases. We restrict ourselves to one-dimensional problems because of the inherent difficulties for generalizing persistence in dimensions greater than one [40-43] which, in turn, impedes obtaining higher-dimensional TEs from the persistent random walk formalism except in two dimensions $[44,45]$ or asymptotically [46]. Higher dimensional FTE's will be the object of future work (see, nonetheless, the end of Sec. VII for an additional and brief discussion on this point).

There have been, to our knowledge, few attempts to derive (or, at least, justify on physical grounds) the FTE. During the last decade a number of works have appeared in the mathematics literature, particularly those of Orshinger and collaborators [47-49], analyzing mathematical and other formal properties of the FTE. However, the fractional equation is set in an ad hoc fashion by replacing the ordinary derivatives that appear in the TE by fractional derivatives which, in addition, may be of various types [49]. Following this way the standard form of the FTE in one dimension reads

$$
\frac{\partial^{2 \alpha} p}{\partial t^{2 \alpha}}+2 \lambda \frac{\partial^{\alpha} p}{\partial t^{\alpha}}=v^{2} \frac{\partial^{2 \gamma} p}{\partial x^{2 \gamma}},
$$


where $0<\alpha \leqslant 1,0<\gamma \leqslant 1$, and $\lambda>0$ and $v$ are given parameters. Equation (10) is the space-time FTE. The particular case $\gamma=1$ is called the time-fractional TE, while $\alpha=1$ corresponds to the space-fractional TE.

One attempt to give physical grounds for the time-fractional TE was the work of Compte and Metzler [50,51] (see also Ref. [52]) who starting from Cattaneo's equation (a modification of Fick's law accounting for noninstantaneous diffusions [8,9]) proposed three different candidates for the one-dimensional time-fractional TE. One of them, having the standard form given in Eq. (10), is derived from the CTRW formalism applied to the probability flux and followed by the assumption of a Gaussian distribution for jump lengths $g(x)$ [50]. We will undertake here a more general approach based on the persistent random walk which results in a space-time FTE agreeing with the standard form given above.

The paper is organized as follows. In Sec. II we review the persistent random walk in continuous time which allows for a direct derivation of the ordinary TE from the random walk picture. In Sec. III we generalize the formalism to include fractional motion, and in Sec. VI we apply such a generalization for obtaining the space-time FTE. In Sec. V we get the exact expression for the characteristic function of the space-time fractional process. In Sec. VI we analyze in detail the time-fractional TE, obtain some asymptotic approximations for the probability density function, and study the mean-square deviation. Some concluding remarks are in Sec. VII.

\section{A CONTINUOUS TIME GENERALIZATION OF THE PERSISTENT RANDOM WALK}

The persistent random walk (PRW) is an example of a random walk with internal states. It is one of the simplest models allowing one to incorporate a property analogous to momentum within the framework of diffusion theory $[15,53,54]$.

The standard analysis starts from a random walk on a onedimensional lattice in discrete time and then assumes that at each node of the lattice the probability that the walker keeps the direction it took in the previous step is equal to $p$ and that the probability the walker reverses direction is $q=1-p$. In general, successive steps are correlated, but when $p=1 / 2$ the PRW reduces to the ordinary random walk with uncorrelated steps. In the diffusive limit, and in the absence of any external field driving the walker, the PDF for the displacement at time $t$ satisfies the one-dimensional TE [15]

$$
\frac{\partial^{2} p}{\partial t^{2}}+\frac{1}{T} \frac{\partial p}{\partial t}=c^{2} \frac{\partial^{2} p}{\partial x^{2}},
$$

with initial conditions

$$
p(x, 0)=\delta(x),\left.\quad \frac{\partial p(x, t)}{\partial x}\right|_{t=0}=0 .
$$

The parameters $T>0$ and $c$ have dimensions of time and velocity, respectively. They are usually constant even though they can also be functions of position and time [55].

Some years ago we developed a continuous time generalization of the PRW (hereafter referred to as CTPRW) in one dimension [13]. We next summarize its main traits.
In one-dimensional PRWs the notion of persistence is incorporated into the analysis by assuming that the random walker can be in one of two states, meaning that the walker moves to the left (plus state) or to the right (minus state). In the continuous time formulation, the duration and length of each step, which we call sojourn, are not fixed but random.

Let us denote by $\psi_{ \pm}(t)$ the PDFs for the time span of each sojourn in the plus or minus state. The probability that the duration of a given sojourn is greater than $t$ will thus be given by

$$
\Psi_{ \pm}(t)=\int_{t}^{\infty} \psi_{ \pm}\left(t^{\prime}\right) d t^{\prime} .
$$

Let us further denote by $f_{+}(x, t)\left[f_{-}(x, t)\right]$ the PDF for the displacement, $X(t)$, of the random walker while in the plus [minus] state. With these densities we define the composite functions

$$
h_{ \pm}(x, t)=f_{ \pm}(x, t) \psi_{ \pm}(t)
$$

and

$$
H_{ \pm}(x, t)=f_{ \pm}(x, t) \Psi_{ \pm}(t) .
$$

Thus $h_{ \pm}(x, t)$ is the joint density for the displacement in a single sojourn in the plus (or minus) state to be equal to $x$ and for the sojourn duration to equal $t$. Analogously, $H_{ \pm}(x, t)$ is the probability density for the displacement to be $x$ in a single sojourn when the total sojourn time in that state is greater than $t$.

We also assume that the random walker is initially at $x=0$ and that $t=0$ marks the beginning of a sojourn in one of the two states, the particular state being chosen with equal probability.

The main goal of the formalism is to know the PDF of the process defined by

$$
p(x, t) d x=\operatorname{Prob}\{x<X(t)<x+d x\} .
$$

Since at any time the process can be in the plus or minus state and both events are mutually exclusive, the PDF can be written as

$$
p(x, t)=p_{+}(x, t)+p_{-}(x, t),
$$

where, for example, $p_{+}(x, t)$ is the PDF for the displacement to be $x$ at time $t$ and for the internal state to be the plus state. In order to proceed further we need two intermediate functions denoted by $\rho_{ \pm}(x, t)$ where

$$
\begin{aligned}
\rho_{ \pm}(x, t) d x d t= & \operatorname{Prob}\{\text { a sojourn in the plus [minus] } \\
& \text { state ends in }(t, t+d t), x<X(t)<x+d x\} .
\end{aligned}
$$

Note that these joint densities describe the state of the process at the regeneration points where the internal state changes. A standard renewal argument readily leads to the following set of coupled integral equation for these intermediate functions:

$$
\begin{aligned}
\rho_{ \pm}(x, t)= & h_{ \pm}(x, t) / 2 \\
& +\int_{-\infty}^{\infty} d x^{\prime} \int_{0}^{t} h_{ \pm}\left(x-x^{\prime}, t-t^{\prime}\right) \rho_{\mp}\left(x^{\prime}, t^{\prime}\right) d t^{\prime} .
\end{aligned}
$$

Indeed, when a sojourn in an occurrence of the plus [minus] state ends at $x$ at time $t$, an event represented by $\rho_{ \pm}(x, t)$, it 
is either the end of the very first sojourn, accounting for the term $h_{ \pm}(x, t)$, or else a sojourn in the minus [plus] state ended at time $t^{\prime}$ where $0<t^{\prime}<t$ with the displacement being $x^{\prime}$ and the subsequent sojourn in the plus (minus) state lasted for a time $t-t^{\prime}$, the displacement during that time being equal to $x-x^{\prime}$. Note that a similar reasoning applies to the PDFs $p_{ \pm}(x, t)$ by replacing the $h$ 's by $H$ 's. We thus have

$$
\begin{aligned}
p_{ \pm}(x, t)= & H_{ \pm}(x, t) / 2 \\
& +\int_{-\infty}^{\infty} d x^{\prime} \int_{0}^{t} H_{ \pm}\left(x-x^{\prime}, t-t^{\prime}\right) \rho_{\mp}\left(x^{\prime}, t^{\prime}\right) d t^{\prime} .
\end{aligned}
$$

These equations can be easily solved using the FourierLaplace transform:

$$
\hat{\tilde{\rho}}_{ \pm}(\omega, s)=\int_{-\infty}^{\infty} e^{-i \omega x} d x \int_{0}^{t} e^{-s t} \rho_{ \pm}(x, t) d t .
$$

The joint transform of Eq. (17) leads to the set of two algebraic equations

$$
\hat{\tilde{\rho}}_{ \pm}(\omega, s)=\left[\frac{1}{2}+\hat{\tilde{\rho}}_{\mp}(\omega, s)\right] \hat{\tilde{h}}_{ \pm}(\omega, s),
$$

whose solution is

$$
\hat{\tilde{\rho}}_{ \pm}(\omega, s)=\frac{\left[1+\hat{\tilde{h}}_{\mp}(\omega, s)\right] \hat{\tilde{h}}_{ \pm}(\omega, s)}{2\left[1-\hat{\tilde{h}}_{+}(\omega, s)\right.},
$$

and the joint transform $\hat{\tilde{p}}(\omega, s)$ of the total PDF reads [cf. Eqs. (16) and (18)]

$$
\hat{\tilde{p}}(\omega, s)=\frac{\hat{\tilde{H}}_{+}(\omega, s)\left[1+\hat{\tilde{h}}_{-}(\omega, s)\right]+\hat{\tilde{H}}_{-}(\omega, s)\left[1+\hat{\tilde{h}}_{+}(\omega, s)\right]}{2\left[1-\hat{\tilde{h}}_{+}(\omega, s) \hat{\tilde{h}}_{-}(\omega, s)\right]} .
$$

This constitutes a generalization of the Montroll-Weiss equation for the CTPRW, and it is the starting point of our subsequent development.

\section{FRACTIONAL PERSISTENT RANDOM WALK IN CONTINUOUS TIME}

As stated in Sec. I, our main objective is to derive, from the persistent random walk picture, the FTE in one dimension. To this end, we will first generalize the PRW to include a fractional version of it.

Let us start by a short review on the probability distribution of the continuous time walk that is the direct generalization of the lattice picture of the PRW. We refer the reader to Ref. [13] for further information.

Recall that in the lattice picture of the PRW the probability that the random walker makes a transition to a neighboring node in a given direction given that the last transition was a step in the opposite direction is $1-p$. Thus the probability that in a sequence of $n$ steps there are $n$ changes of direction is $(1-p)^{n}$ and the probability that there will be a sequence of $n$ consecutive steps in the same direction is $p^{n}$. The continuous analog of this result is found by choosing $\psi_{ \pm}(t)$ to have the isotropic property, $\psi_{+}(t)=\psi_{-}(t)$, and the exponential form

$$
\psi_{ \pm}(t)=\lambda e^{-\lambda t}
$$

where $\lambda^{-1}$ is the average duration of a sojourn. In this case the instants of time, $t_{1}, t_{2}, t_{3}, \ldots$, where changes of direction occur (the so-called regeneration times) are distributed according to the Poisson distribution [56].

Moreover, in the lattice picture the displacement is fixed and given by the length of a single site. The continuum analog of this requirement implies that the displacement in a single sojourn is exactly proportional to the time spent in that sojourn. In other words, the functions $f_{ \pm}(x, t)$ correspond to deterministic and uniform motion,

$$
f_{ \pm}(x, t)=\delta(x \mp c t),
$$

where $c$ is the speed of the walker.

Two observations are in order. First, any force driving the random walker would result in a nonlinear argument in the delta function, and, second, the motion of the walker in a given sojourn could also be random. In such a case $f_{ \pm}(x, t)$ would be given by an appropriate PDF other than the delta distribution given in Eq. (22).

With these forms of $\psi_{ \pm}(t)$ and $f_{ \pm}(x, t)$ the transforms of $h_{ \pm}(x, t)$ and $H_{ \pm}(x, t)$ are easily found to be

$$
\hat{\tilde{h}}_{ \pm}(\omega, s)=\frac{\lambda}{\lambda+s \pm i c \omega}, \quad \hat{\tilde{H}}_{ \pm}(\omega, s)=\frac{1}{\lambda+s \pm i c \omega} .
$$

We observe that in the fluid limit $(\omega, s \rightarrow 0)$ functions $\hat{\tilde{h}}_{ \pm}(\omega, s)$ behave as

$$
\hat{\tilde{h}}_{ \pm}(\omega, s)=1-(1 / \lambda) s \mp i(c / \lambda) \omega \cdots
$$

and with a similar expansion for $\hat{\tilde{H}}_{ \pm}(\omega, s)$.

Inserting Eq. (23) into Eq. (20) yields

$$
\hat{\tilde{p}}(\omega, s)=\frac{2 \lambda+s}{s^{2}+2 \lambda s+c^{2} \omega^{2}},
$$

which determines the probability distribution of the random walk that is the continuous time version of the PRW in the lattice, in other words, when sojourn duration densities $\psi_{ \pm}(t)$ and displacement densities $f_{ \pm}(x, t)$ are given, respectively, by Eqs. (21) and (22).

Before proceeding to the generalization of Eq. (25) to include fractional walks, let us note that the partial differential equation satisfied by $p(x, t)$ may be found by multiplying both sides of Eq. (25) by the denominator and proceeding afterwards to the Fourier-Laplace inversion. Assuming the initial conditions given in Eq. (12), the Fourier-Laplace inversion results in the one-dimensional TE [13]

$$
\frac{\partial^{2} p}{\partial t^{2}}+2 \lambda \frac{\partial p}{\partial t}=c^{2} \frac{\partial^{2} p}{\partial x^{2}}
$$

In order to derive a FTE out of the CTPRW picture, we will first propose a fractional generalization of the CTPRW. To this end we follow the footsteps of the derivation of the fractional generalization of the CTRW which led to the FDE (see Sec. I). Thus, mimicking Eq. (24) we assume the following expansion for the Fourier-Laplace transform of the sojourn's joint densities $h_{ \pm}(\omega, s)$ in the fluid limit:

$$
\hat{\tilde{h}}_{ \pm}(\omega, s)=1-(T s)^{\alpha} \mp i(L \omega)^{\gamma} \ldots
$$


$(s, \omega \rightarrow 0)$, where $0<\alpha \leqslant 1,0<\gamma \leqslant 1$ and $T>0$ and $L>0$ are arbitrary parameters. $T$ sets a characteristic time and $L$ a characteristic length.

We note that within the same degree of approximation, that is, assuming $s$ and $\omega$ small, the expansion (27) is equivalent to

$$
\hat{\tilde{h}}_{ \pm}(\omega, s) \simeq \frac{1}{1+(T s)^{\alpha} \pm i(L \omega)^{\gamma}}
$$

$(s, \omega \rightarrow 0)$.

In order to proceed further we also need to assume a fluidlimit approximation for $\hat{\tilde{H}}_{ \pm}(\omega, s)$ consistent with Eq. (28). Let us recall that $h_{ \pm}(x, t)$ are the joint densities for the length and duration of sojourns in the plus or minus state. Therefore, their time marginal densities are

$$
\int_{-\infty}^{\infty} h_{ \pm}(x, t) d x=\psi_{ \pm}(t),
$$

where $\psi_{ \pm}(t)$ are the PDFs for sojourn duration in each state. Correspondingly

$$
\int_{-\infty}^{\infty} H_{ \pm}(x, t) d x=\Psi_{ \pm}(t)
$$

where $\Psi_{ \pm}(t)$ is given in Eq. (13). In the Fourier-Laplace space these conditions read

$$
\hat{\tilde{h}}_{ \pm}(\omega=0, s)=\hat{\psi}_{ \pm}(s), \quad \text { and } \quad \hat{\tilde{H}}_{ \pm}(\omega=0, s)=\hat{\Psi}_{ \pm}(s),
$$

but from Eq. (13) we see that $\hat{\Psi}_{ \pm}(s)=\left[1-\hat{\psi}_{ \pm}(s)\right] / s$, hence

$$
\hat{\tilde{H}}_{ \pm}(\omega=0, s)=\frac{1}{s}\left[1-\hat{\tilde{h}}_{ \pm}(\omega=0, s)\right] .
$$

Inserting Eq. (28) into this expression yields

$$
\hat{\tilde{H}}_{ \pm}(\omega=0, s) \simeq \frac{T(T s)^{\alpha-1}}{1+(T s)^{\alpha}},
$$

which leads us to assume

$$
\hat{\tilde{H}}_{ \pm}(\omega, s) \simeq \frac{T(T s)^{\alpha-1}}{1+(T s)^{\alpha} \pm i(L \omega)^{\gamma}},
$$

as $s \rightarrow 0$ and $\omega \rightarrow 0$. Let us stress that this is a conjecture, because the numerator of Eq. (29) might have depended on $\omega$ as well.

Substituting Eqs. (28) and (29) into Eq. (20) and simple algebra yield

$$
\hat{\tilde{p}}(\omega, s)=\frac{T(T s)^{\alpha-1}\left[(T s)^{\alpha}+2\right]}{(T s)^{2 \alpha}+2(T s)^{\alpha}+(L \omega)^{2 \gamma}} .
$$

Dividing numerator and denominator by $T^{2 \alpha}$ we get the neater form:

$$
\hat{\tilde{p}}(\omega, s)=\frac{s^{\alpha-1}\left(s^{\alpha}+2 \lambda\right)}{s^{2 \alpha}+2 \lambda s^{\alpha}+v^{2} \omega^{2 \gamma}},
$$

where

$$
\lambda \equiv 1 / T^{\alpha}, \quad v \equiv L^{\gamma} / T^{\alpha} .
$$

Equation (31) determines the probability distribution of the fractional persistent random walk. It constitutes one of the main results of the paper, and it is the starting point of our further analysis.

\section{SPACE-TIME FRACTIONAL TELEGRAPHER'S EQUATION}

We now proceed to derive from the CTPRW picture the onedimensional FTE. To this end we first need some definitions.

The Caputo fractional derivative of order $\beta>0$ of a function $\phi(t)$ is defined by the functional [31-36]

$$
\frac{\partial^{\beta} \phi(t)}{\partial t^{\beta}}= \begin{cases}\frac{1}{\Gamma(n-\beta)} \int_{0}^{t} \frac{\phi^{(n)}\left(t^{\prime}\right) d t^{\prime}}{\left(t-t^{\prime}\right)^{1+\beta-n}}, & n-1<\beta<n, \\ \phi^{(n)}(t), & \beta=n\end{cases}
$$

( $n=1,2,3, \ldots)$. Using this definition the Laplace transform of the Caputo derivative is found to be [32]

$$
\mathcal{L}\left\{\frac{\partial^{\beta} \phi(t)}{\partial t^{\beta}}\right\}=s^{\beta} \hat{\phi}(s)-s^{\beta-1} \phi(0)-\sum_{j=1}^{n-1} s^{\beta-1-j} \phi^{(j)}(0)
$$

$(n=1,2,3, \ldots ; n-1<\beta<n)$, where $\mathcal{L}\{\cdot\}$ stands for the Laplace transform and $\hat{\phi}(s)=\mathcal{L}\{\phi(t)\}$.

The second kind of fractional derivative we need to define is the Riesz-Feller fractional derivative of order $\beta(0<\beta \leqslant 2)$ of a function $g(x)$ such that $g(x) \rightarrow 0$ as $x \rightarrow \pm \infty$. There are several equivalent ways to define it [32], although one of the simplest and most operative definitions is obtained using Fourier analysis. We thus define [36]

$$
\frac{\partial^{\beta} g(x)}{\partial|x|^{\beta}}=\mathcal{F}^{-1}\left\{-|\omega|^{\beta} \tilde{g}(\omega)\right\}
$$

$(0<\beta \leqslant 2)$, where $\mathcal{F}^{-1}\{\cdot\}$ stands for the inverse Fourier transform, and

$$
\tilde{g}(\omega)=\int_{-\infty}^{\infty} e^{i \omega x} g(x) d x
$$

is the direct transform

Let us return to the derivation of the FTE. The starting point is Eq. (31), which we write in the form

$$
\left(s^{2 \alpha}+2 \lambda s^{\alpha}+v^{2} \omega^{2 \gamma}\right) \hat{\tilde{p}}(\omega, s)=s^{2 \alpha-1}+2 \lambda s^{\alpha-1} .
$$

Fourier inverting this equation and taking into account the definition of the Riesz-Feller derivative [Eq. (35)], we get

$$
\left(s^{2 \alpha}+2 \lambda s^{\alpha}-v^{2} \frac{\partial^{2 \gamma}}{\partial x^{2 \gamma}}\right) \hat{p}(x, s)=\left(s^{2 \alpha-1}+2 \lambda s^{\alpha-1}\right) \delta(x),
$$

where $\hat{p}(x, s)$ is the Laplace transform of the PDF. We rewrite this last expression as

$$
\begin{aligned}
& s^{2 \alpha} \hat{p}(x, s)-s^{2 \alpha-1} \delta(x) \\
& \quad+2 \lambda\left[s^{\alpha} \hat{p}(x, s)-s^{\alpha-1} \delta(x)\right]=v^{2} \frac{\partial^{2 \gamma} \hat{p}}{\partial x^{2 \gamma}} .
\end{aligned}
$$

In order to invert Eq. (36), we first evaluate the Laplace transforms of the fractional derivatives $\partial^{\alpha} p / \partial t^{\alpha}$ and $\partial^{2 \alpha} p / \partial t^{2 \alpha}$ using Eq. (34). We must distinguish the cases $\beta=\alpha$ and $\beta=2 \alpha$. 
(i) Set $\beta=\alpha$ in Eq. (34), and since $0<\alpha \leqslant 1$ we see that $n=1$. Hence

$$
\mathcal{L}\left\{\frac{\partial^{\alpha} p(x, t)}{\partial t^{\alpha}}\right\}=s^{\alpha} \hat{p}(x, s)-s^{\alpha-1} p(x, 0) .
$$

But $p(x, 0)=\delta(x)$ [cf. Eq. (12)] so that

$$
\frac{\partial^{\alpha} p}{\partial t^{\alpha}}=\mathcal{L}^{-1}\left\{s^{\alpha} \hat{p}(x, s)-s^{\alpha-1} \delta(x)\right\} .
$$

(ii) When $\beta=2 \alpha(0<\alpha \leqslant 1)$ we need to separate the cases: (a) $0<\alpha \leqslant 1 / 2$ and (b) $1 / 2<\alpha \leqslant 1$. For case (a) we have $0<2 \alpha \leqslant 1$, and from Eq. (34) with $\beta=2 \alpha$ and $n=1$, we obtain

$$
\mathcal{L}\left\{\frac{\partial^{2 \alpha} p(x, t)}{\partial t^{\alpha}}\right\}=s^{2 \alpha} \hat{p}(x, s)-s^{2 \alpha-1} \delta(x) .
$$

In case (b) we have $1<2 \alpha \leqslant 2$, and from Eq. (34) with $n=2$ we write

$$
\begin{aligned}
& \mathcal{L}\left\{\frac{\partial^{2 \alpha} p(x, t)}{\partial t^{\alpha}}\right\} \\
& \quad=s^{2 \alpha} \hat{p}(x, s)-s^{2 \alpha-1} \delta(x)-\left.s^{2(\alpha-1)} \frac{\partial p(x, t)}{\partial t}\right|_{t=0} .
\end{aligned}
$$

Since $\partial p /\left.\partial t\right|_{t=0}=0$ [cf. Eq. (12)] we see that this case coincides with case (a) above. Therefore,

$$
\frac{\partial^{2 \alpha} p}{\partial t^{2 \alpha}}=\mathcal{L}^{-1}\left\{s^{2 \alpha} \hat{p}(x, s)-s^{2 \alpha-1} \delta(x)\right\} .
$$

The Laplace inversion of Eq. (36) and the use of Eqs. (37) and (38) yield the space-time FTE:

$$
\frac{\partial^{2 \alpha} p}{\partial t^{2 \alpha}}+2 \lambda \frac{\partial^{\alpha} p}{\partial t^{\alpha}}=v^{2} \frac{\partial^{2 \gamma} p}{\partial x^{2 \gamma}} .
$$

From Eq. (39) we can easily obtain the fractional diffusion equation in one dimension. Indeed, letting $\lambda \rightarrow \infty$, which is equivalent to assume that $T \rightarrow 0$ [cf. Eq. (32)], and also letting $v \rightarrow \infty$ such that

$$
\frac{v^{2}}{2 \lambda}=\frac{L^{2 \gamma}}{2 T^{\alpha}} \rightarrow D \text { (finite), }
$$

we get the one-dimensional FDE [cf. Eq. (4)]

$$
\frac{\partial^{\alpha} p}{\partial t^{\alpha}}=D \frac{\partial^{2 \gamma} p}{\partial x^{2 \gamma}} .
$$

Let us remark that even for any finite value of the parameter $\lambda$, the FDE is the asymptotic (in time) limit of the FTE. Indeed, by passing to the limit $s \rightarrow 0$ in Eq. (31) the small $s$ approximation to $\hat{\tilde{p}}(\omega, s)$ is found to be

$$
\hat{\tilde{p}}(\omega, s) \simeq \frac{s^{\alpha-1}}{s^{\alpha}+\left(v^{2} / 2 \lambda\right) \omega^{2 \gamma}},
$$

which after Fourier-Laplace inversion yields Eq. (40).

The FTE (39) also contains the fractional wave equation (FWE) as a special case. Thus, letting $\lambda \rightarrow 0$ (i.e., $T \rightarrow \infty$ and at the same time $L \rightarrow \infty$ such that $v=L^{\gamma} / T^{\alpha}$ remains finite) from Eq. (39) we get

$$
\frac{\partial^{2 \alpha} p}{\partial t^{2 \alpha}}=v^{2} \frac{\partial^{2 \gamma} p}{\partial x^{2 \gamma}} .
$$

Note that when $\alpha=1 / 2$ and $\gamma=1$ this equation reduces to the ordinary diffusion equation. In this regard Mainardi's expression, "fractional diffusion-wave equation," is more accurate than FWE [33].

On the other hand, Eq. (42) is the small time limit of the FTE regardless of the value of $\lambda$. Indeed, the limit $s \rightarrow \infty$ in Eq. (31) yields

$$
\hat{\tilde{p}}(\omega, s) \simeq \frac{s^{2 \alpha-1}}{s^{2 \alpha}+v^{2} \omega^{2 \gamma}},
$$

and the Fourier-Laplace inversion results in Eq. (42).

We may, therefore, conclude from the preceding analysis that the FTE combines two different dynamics: one, at small times, representing fractional wavelike behavior, and another one which at large times enhances fractional diffusion-like behavior. This constitutes a generalization of the dual character of the ordinary TE between waves and diffusions (see Sec. I).

\section{CHARACTERISTIC FUNCTION}

We next obtain the characteristic function $\tilde{p}(\omega, t)$ [i.e., the Fourier transform of the PDF $p(x, t)]$ of the spacetime fractional telegraph process by performing the Laplace inversion of the function $\hat{\tilde{p}}(\omega, s)$. We thus start off with Eq. (31), which after some elementary manipulations can be written as

$$
\hat{\tilde{p}}(\omega, s)=\frac{s^{\alpha-1}}{2 \eta(\omega)}\left[\frac{\lambda+\eta(\omega)}{s^{\alpha}+\lambda-\eta(\omega)}-\frac{\lambda-\eta(\omega)}{s^{\alpha}+\lambda+\eta(\omega)}\right],
$$

where

$$
\eta(\omega)=\sqrt{\lambda^{2}-\left(v \omega^{\gamma}\right)^{2}} .
$$

Further manipulations yield

$$
\begin{aligned}
\frac{s^{\alpha-1}}{s^{\alpha}+\lambda \pm \eta(\omega)} & =\frac{1 / s}{1+[\lambda \pm \eta(\omega)] / s^{\alpha}} \\
& =\sum_{n=0}^{\infty}(-1)^{n} \frac{[\lambda \pm \eta(\omega)]^{n}}{s^{1+n \alpha}} .
\end{aligned}
$$

Since [57]

$$
\mathcal{L}^{-1}\left\{\frac{1}{s^{1+n \alpha}}\right\}=\frac{t^{n \alpha}}{\Gamma(1+n \alpha)},
$$

we have

$$
\begin{aligned}
\mathcal{L}^{-1}\left\{\frac{s^{\alpha-1}}{s^{\alpha}+\lambda \pm \eta(\omega)}\right\} & =\sum_{n=0}^{\infty}(-1)^{n} \frac{\left([\lambda \pm \eta(\omega)] t^{\alpha}\right)^{n}}{\Gamma(1+n \alpha)} \\
& =E_{\alpha}\left(-[\lambda \pm \eta(\omega)] t^{\alpha}\right),
\end{aligned}
$$

where $E_{\alpha}(\cdot)$ is the Mittag-Leffler function [58]

$$
E_{\alpha}(z)=\sum_{n=0}^{\infty} \frac{z^{n}}{\Gamma(1+n \alpha)} .
$$

Taking the inverse Laplace transform of Eq. (44) and using the above intermediate results we finally obtain the characteristic function of the space-time fractional process

$$
\begin{aligned}
\tilde{p}(\omega, t)= & \frac{1}{2 \eta(\omega)}\left\{[\lambda+\eta(\omega)] \mathrm{E}_{\alpha}\left(-[\lambda-\eta(\omega)] t^{\alpha}\right)\right. \\
& \left.-[\lambda-\eta(\omega)] \mathrm{E}_{\alpha}\left(-[\lambda+\eta(\omega)] t^{\alpha}\right)\right\} .
\end{aligned}
$$


In the wavelike limit $\lambda \rightarrow 0$ the FTE (39) reduces to the FWE (42). In this case [cf. Eq. (45)]

$$
\eta(\omega)=i v|\omega|^{\gamma},
$$

and the characteristic function reads

$$
\tilde{p}(\omega, t)=\frac{1}{2}\left[\mathrm{E}_{\alpha}\left(-i v|\omega|^{\gamma} t^{\alpha}\right)+\mathrm{E}_{\alpha}\left(i v|\omega|^{\gamma} t^{\alpha}\right)\right],
$$

a solution already obtained by Mainardi [33]. When $\alpha=1$ and since $\mathrm{E}_{1}(z)=e^{z}$ [cf. Eq. (46)], we have

$$
\tilde{p}(\omega, t)=\frac{1}{2}\left[e^{-i v|\omega|^{\gamma} t}+e^{i v|\omega|^{\gamma} t}\right] .
$$

Finally, for the case $\gamma=1$, which corresponds to the regular wave equation, we can invert the characteristic function and get the expected result for the propagation of an undisturbed wave:

$$
p(x, t)=\frac{1}{2}[\delta(x+v t)+\delta(x-v t)] .
$$

In the diffusion-like limit $\lambda \rightarrow \infty$ and $v^{2} / \lambda \rightarrow D$ (finite), and from Eq. (45) we see that

$$
\frac{\lambda}{\eta(\omega)}=\frac{1}{\sqrt{1-\left(v \omega^{\gamma} / \lambda\right)^{2}}} \rightarrow 1
$$

and

$$
\lambda-\eta(\omega)=\frac{\left(v^{2} / \lambda\right) \omega^{2 \gamma}}{1+\sqrt{1-\left(v \omega^{\gamma} / \lambda\right)^{2}}} \rightarrow D \omega^{2 \gamma} .
$$

From Eq. (47) we get

$$
\tilde{p}(\omega, t)=\mathrm{E}_{\alpha}\left[-D t^{\alpha} \omega^{2 \gamma}\right],
$$

a well-known result which corresponds to a Lévy density with fractional time $[20,22]$. When $\alpha=1$ (no fractional time but fractional space) this result reduces to the ordinary Lévy distribution with zero mean,

$$
\tilde{p}(\omega, t)=e^{-D t \omega^{2 \gamma}} .
$$

\section{TIME-FRACTIONAL TELEGRAPHER'S EQUATION}

The time-fractional TE is a particular case of the space-time FTE (39) when $\gamma=1$ :

$$
\frac{\partial^{2 \alpha} p}{\partial t^{2 \alpha}}+2 \lambda \frac{\partial^{\alpha} p}{\partial t^{\alpha}}=v^{2} \frac{\partial^{2} p}{\partial x^{2}} .
$$

In the Fourier-Laplace space the solution to this equation with initial conditions (12) is given by [cf. Eq. (31)]

$$
\hat{\tilde{p}}(\omega, s)=\frac{s^{\alpha-1}\left(s^{\alpha}+2 \lambda\right)}{s^{2 \alpha}+2 \lambda s^{\alpha}+v^{2} \omega^{2}} .
$$

The Fourier inversion of this expression is now possible. Indeed, recalling the Fourier transform

$$
\mathcal{F}^{-1}\left\{\frac{a}{b+v^{2} \omega^{2}}\right\}=\frac{a}{2 v b^{1 / 2}} e^{-|x| b^{1 / 2} / v},
$$

we get

$$
\hat{p}(x, s)=\frac{1}{2 v s} \sqrt{s^{2 \alpha}+2 \lambda s^{\alpha}} \exp \left\{-\frac{|x|}{v} \sqrt{s^{2 \alpha}+2 \lambda s^{\alpha}}\right\} .
$$

For $\alpha=1$, Eq. (51) reduces to the ordinary TE (11), and the Laplace transform (53) can be inverted exactly with the well-known result [7]

$$
\begin{aligned}
p(x, t)= & \frac{1}{2}\{\delta(x-v t)+\delta(x+v t) \\
& \left.+\frac{\lambda}{2 v} \Theta(v t-|x|)\left[I_{0}(\rho)+\frac{\lambda}{\rho} I_{1}(\rho)\right]\right\},
\end{aligned}
$$

where $\delta(x \mp v t)$ are two $\delta$ pulses moving in opposite directions, $\Theta(\cdot)$ is the Heaviside step function, the variable $\rho$ is

$$
\rho=\frac{\lambda}{v} \sqrt{v^{2} t^{2}-x^{2}}
$$

and $I_{0}(\rho)$ and $I_{1}(\rho)$ are modified Bessel functions.

For the fractional case when $\alpha \neq 1$, the exact analytical inversion of Eq. (53) seems to be very involved, possibly beyond reach. We can, nonetheless, obtain approximate solutions for large and small values of time using Tauberian theorems which relate the small $s$ [large $s$ ] behavior of $\hat{p}(x, s)$ with the large $t$ [small $t$ ] behavior of $p(x, t)$ [59].

Noting that

$$
\sqrt{s^{2 \alpha}+2 \lambda s^{\alpha}}=\left(2 \lambda s^{\alpha}\right)^{1 / 2}\left[1+O\left(\frac{s^{\alpha}}{\lambda}\right)\right],
$$

we can approximate Eq. (53) for small values of $s$ (specifically when $s \ll \lambda^{1 / \alpha}$ ) by

$$
\hat{p}(x, s) \simeq \frac{(\lambda / 2)^{1 / 2}}{v s^{1-\alpha / 2}} e^{-|x|\left(2 \lambda s^{\alpha}\right)^{1 / 2} / v} .
$$

Let us incidentally note that Eq. (56) corresponds to the Laplace transform of the exact solution to the FDE

$$
\frac{\partial^{\alpha} p}{\partial t^{\alpha}}=D \frac{\partial^{2} p}{\partial x^{2}},
$$

obtained by passing Eq. (51) to the limit $\lambda \rightarrow \infty$ with $v \rightarrow \infty$ and such that $D=v^{2} / 2 \lambda$ is finite. This is a fact already pointed out above and constitutes the generalization of the central limit theorem for fractional processes.

The Laplace inversion of Eq. (56) leads to the long-time solution to the time-fractional TE (51). As shown in the Appendix this inversion results in the following asymptotic approximation valid as $t \gg \lambda^{1 / \alpha}$ :

$$
p(x, t) \simeq \frac{\sqrt{\lambda / 2}}{v t^{\alpha / 2}} M_{\alpha / 2}\left(\frac{|x| \sqrt{\lambda / 2}}{v t^{\alpha / 2}}\right),
$$

where $M_{\alpha / 2}(\cdot)$ is the Mainardi function defined by the power series $[33,60]$

$$
M_{\beta}(z)=\sum_{n=0}^{\infty} \frac{(-1)^{n} z^{n}}{n ! \Gamma(-\beta n+1-\beta)} .
$$

It can be proved that $M_{\beta}(z)$ is an entire function for $0<$ $\beta<1$ [33]. It is a special case of the Wright function [58,60], which is, in turn, closely related to the rather cumbersome Fox function, the latter frequently used in the anomalous diffusion literature [20].

Let us note that, after the replacement $v^{2} / 2 \lambda \rightarrow D$, the asymptotic expression (58) becomes the exact solution to the FDE (57) [33]. 
We next analyze the large $s$ behavior of Eq. (53), which corresponds to the small time behavior of $p(x, t)$. For large values of $s$ such that $s \gg \lambda^{1 / \alpha}$, we may write

$$
\sqrt{s^{2 \alpha}+2 \lambda s^{\alpha}}=s^{\alpha}\left[1+O\left(\frac{\lambda}{s^{\alpha}}\right)\right],
$$

and when $s \gg \lambda^{1 / \alpha}$ we can approximate Eq. (53) by

$$
\hat{p}(x, s) \simeq \frac{s^{\alpha-1}}{2 v} e^{-|x| s^{\alpha} / v} .
$$

Again, this expression corresponds to the Laplace transform of the exact solution to the fractional wave-diffusion equation

$$
\frac{\partial^{2 \alpha} p}{\partial t^{2 \alpha}}=v^{2} \frac{\partial^{2} p}{\partial x^{2}}
$$

obtained by passing Eq. (51) to the limit $\lambda \rightarrow 0$ with $v$ finite.

We return to Eq. (60), and expanding the exponential we get $\left(s \gg \lambda^{1 / \alpha}\right)$

$$
\hat{p}(x, s) \simeq \frac{1}{2 v} \sum_{n=0}^{\infty} \frac{(-1)^{n}}{n !} \frac{(|x| / v)^{n}}{s^{1-(n+1) \alpha}} .
$$

After using Eq. (A1) of the Appendix, the Laplace inversion of this equation yields

$$
p(x, t) \simeq \frac{1}{2 v t^{\alpha}} \sum_{n=0}^{\infty} \frac{\left(-|x| / v t^{\alpha}\right)^{n}}{n ! \Gamma[1-(n+1) \alpha]},
$$

which, in terms of Mainardi function (59), can be written as

$$
p(x, t) \simeq \frac{1}{2 v t^{\alpha}} M_{\alpha}\left(\frac{|x|}{v t^{\alpha}}\right),
$$

an expression valid for small times such that $t \ll \lambda^{1 / \alpha}$.

Finally we will evaluate the mean square displacement, a basic characteristic of anomalous transport, for the timefractional process described by Eq. (51).

Let us recall that moments $\left\langle X^{n}(t)\right\rangle$ are related to derivatives of the characteristic function $\tilde{p}(\omega, t)$ with respect to $\omega$ evaluated at $\omega=0$. Hence, in terms of the Laplace transform of the characteristic function $\hat{\tilde{p}}(\omega, s)$, we may write

$$
\mathcal{L}\left\{\left\langle X^{n}(t)\right\rangle\right\}=\left.i^{-n} \frac{\partial^{n} \hat{\tilde{p}}(\omega, s)}{\partial \omega^{n}}\right|_{\omega=0} .
$$

Using Eq. (52) we easily see that the first moment is zero while the Laplace transform of the second moment reads

$$
\mathcal{L}\left\{\left\langle X^{2}(t)\right\rangle\right\}=\frac{2 v^{2}}{s^{\alpha+1}\left(s^{\alpha}+2 \lambda\right)} .
$$

Let us proceed to Laplace inversion. Noting that

$$
\begin{aligned}
\frac{1}{s^{\alpha+1}\left(s^{\alpha}+2 \lambda\right)} & =\frac{1}{s^{2 \alpha+1}} \frac{1}{1+2 \lambda s^{-\alpha}} \\
& =\sum_{n=0}^{\infty}(-1)^{n} \frac{(2 \lambda)^{n}}{s^{(n+2) \alpha+1}},
\end{aligned}
$$

and recalling that $\mathcal{L}^{-1}\left\{s^{-\delta}\right\}=t^{\delta-1} / \Gamma(\delta)(\delta>0)$ [57], we get the exact expression for the mean square displacement [48]

$$
\left\langle X^{2}(t)\right\rangle=2 v^{2} t^{2 \alpha} E_{\alpha, 2 \alpha+1}\left(-2 \lambda t^{\alpha}\right)
$$

$(0<\alpha \leqslant 1)$ where $E_{\alpha, \beta}(\cdot)$ is the two-parameter Mittag-Leffler function defined as [58]

$$
E_{\alpha, \beta}(z)=\sum_{n=0}^{\infty} \frac{z^{n}}{\Gamma(n \alpha+\beta)} .
$$

The small time behavior of the mean square displacement is easily obtained by observing from Eq. (65) that for small values of $z, E_{\alpha, \beta}(z)=1 / \Gamma(\beta)+O(z)$. Therefore,

$$
\left\langle X^{2}(t)\right\rangle=\frac{2 v^{2} t^{2 \alpha}}{\Gamma(1+2 \alpha)}\left[1+O\left(\lambda t^{\alpha}\right)\right] .
$$

For the nonfractional TE $\alpha=1$, and we recover the wellknown result that $\left\langle X^{2}(t)\right\rangle \sim t^{2}$ is ballistic at short times. We also observe that when $t \ll \lambda^{1 / \alpha}$, we have subdiffusion if $0<$ $\alpha<1 / 2$, normal diffusion if $\alpha=1 / 2$, and superdiffusion if $1 / 2<\alpha \leqslant 1$.

For getting the large time behavior of the mean square displacement we use the following asymptotic approximation of the two-parameter Mittag-Leffler function [63,64]:

$$
E_{\alpha, \beta}(-x) \sim \sum_{n=1}^{N-1} \frac{(-x)^{-n}}{\Gamma(\beta-n \alpha)}+O\left(\frac{1}{x^{N}}\right),
$$

where $x>0,0<\alpha<1$, and $N=2,3, \ldots$ Therefore,

$$
E_{\alpha, 2 \alpha+1}\left(-2 \lambda t^{\alpha}\right) \sim \frac{t^{-\alpha}}{2 \lambda \Gamma(\alpha+1)}+O\left(\frac{1}{\lambda^{2} t^{2 \alpha}}\right) .
$$

Plugging it into Eq. (64) we get

$$
\left\langle X^{2}(t)\right\rangle \sim \frac{v^{2}}{\lambda \Gamma(\alpha+1)} t^{\alpha}, \quad(t \rightarrow \infty),
$$

and the mean square displacement is always subdiffusive at long times.

We, therefore, conclude that as time progresses there are transitions between two different subdiffusive regimes if $0<$ $\alpha<1 / 2$ but also from superdiffusion to subdiffusion when $1 / 2<\alpha \leqslant 1$. The case $\alpha=1 / 2$ is rather singular, since it goes from normal diffusion to subdiffusion.

\section{CONCLUDING REMARKS}

In this work we have generalized the telegrapher's equation to account for anomalous transport in one dimension. We have derived the space-time fractional telegrapher's equation from the formalism of the persistent random walk in continuous time.

To this end we have had to generalize the persistent random walk to allow for fractional behavior. This is achieved by assuming that the densities governing the evolution of the walk, $h_{+}(x, t)$ and $h_{-}(x, t)$, have in the fluid limit (large times and distances) the form given by Eq. (27) in terms of two exponents $\alpha$ and $\gamma$, both taking values between 0 and 1 . All of this results in the standard form of the FTE given in Eq. (39):

$$
\frac{\partial^{2 \alpha} p}{\partial t^{2 \alpha}}+2 \lambda \frac{\partial^{\alpha} p}{\partial t^{\alpha}}=v^{2} \frac{\partial^{2 \gamma} p}{\partial x^{2 \gamma}} .
$$

For $\lambda \rightarrow 0$ and $v$ finite this equation reduces to the (fractional) diffusion-wave equation (42), and the same happens for small times $\left(t \ll \lambda^{1 / \alpha}\right)$ regardless the values of $\lambda$ and $v$. In the 
opposite case when $\lambda \rightarrow \infty$ and $v \rightarrow \infty$ but $D=v^{2} / 2 \lambda$ finite, or for any value of these parameters but at large times $(t \gg$ $\lambda^{1 / \alpha}$ ), the TE reduces to the (fractional) diffusion equation (40). Therefore, the FTE combines two different dynamics: one of them, governing the process at small times, is given by fractional wave behavior, while at large times the dynamics is ruled by fractional diffusion behavior. This constitutes a generalization of the dual character of the ordinary TE between waves and diffusion.

This dual character is even more apparent for the timefractional equation when $\gamma=1$ and for which only time is fractional [cf. Eq. (51)]. In this case, the mean square displacement exists, and its exact expression is given by Eq. (64) in terms of the two-parameter Mittag-Leffler function. For small and large times the mean square displacement is approximated by

$$
\langle X(t)\rangle \sim t^{2 \alpha},(t \rightarrow 0) ; \quad\langle X(t)\rangle \sim t^{\alpha},(t \rightarrow \infty) .
$$

We thus see that in the course of time, the fractional telegraph process undergoes transitions between different anomalous diffusion regimes. When $0<\alpha<1 / 2$ there is a transition from two different subdiffusive regimes, while if $1 / 2<\alpha<1$ the transition is from superdiffusion to subdiffusion. A relevant fact because generalizes the transition between ballistic motion and normal diffusion shown by the ordinary TE.

We finish this paper with a brief discussion on higher dimensional problems. As we mentioned in Sec. I, higherdimensional TEs are not well founded on higher-dimensional persistent random walks [40-46], a circumstance that hinders possible derivations of a higher-dimensional fractional TE out of some fractional generalization of the persistent random walk as we have done in the one-dimensional case. Let us, however. note that from a purely formal point of view, the ansatz given by Eq. (28) could be extended in an ad hoc manner to higher dimensions:

$$
\hat{\tilde{h}}_{ \pm}(\omega, s) \simeq \frac{1}{1+(T s)^{\alpha} \pm(L|\omega|)^{\gamma}}
$$

$(s,|\omega| \rightarrow 0)$ and with a similar expression for $\hat{\tilde{H}}_{ \pm}(\boldsymbol{\omega}, s)$ [cf. Eq. (29)]. Assuming next that the joint transform of the PDF is given by Eq. (20) and following the same procedure as that of Secs. III and IV we arrive at the higher-dimensional fractional TE for the PDF $p(\mathbf{r}, t)$ :

$$
\frac{\partial^{2 \alpha} p}{\partial t^{2 \alpha}}+2 \lambda \frac{\partial^{\alpha} p}{\partial t^{\alpha}}=v^{2} \nabla^{2 \gamma} p,
$$

where $\lambda$ and $v$ given in Eq. (32) and $\nabla^{2 \gamma}$ is the Riesz-Feller fractional Laplacian.

However, this (formal) derivation is not really based on the continuous-time persistent random walk. It turns out that for the nonfractional case where $\alpha=\gamma=1$, the functions $h_{ \pm}(\mathbf{r}, t)$ resulting from inverting Eq. (69) have an imaginary part, which means that they do not represent a true PDF. Therefore. such a microscopic justification of Eq. (70) based on ansatz (69) is rather dubious. The question of justifying Eq. (70) from a microscopic model is under study.

\section{ACKNOWLEDGMENTS}

The author acknowledges partial financial support from MINECO (Spain) under Contract No. FIS2013-47532-C3-2-P and from Generalitat de Catalunya (Spain) under Contract 2014 SGR 608.

\section{APPENDIX: LONG-TIME SOLUTION OF THE TIME FRACTIONAL TE}

In order to show that Eq. (58) is the Laplace inversion of Eq. (56) we essentially follow Mainardi (Ref. [33]). We thus first prove the inversion formula

$$
\mathcal{L}^{-1}\left\{s^{\delta}\right\}=\frac{t^{-1-\delta}}{\Gamma(-\delta)},
$$

where $\delta \neq 0$ is not a positive integer but otherwise an arbitrary (positive or negative) real number [recall that when $\delta<0$, Eq. (A1) reduces to a well known inversion formula [57]].

In effect, from the definition of Laplace inversion we have

$$
\begin{aligned}
\mathcal{L}^{-1}\left\{s^{\delta}\right\} & =\frac{1}{2 \pi i} \int_{\mathrm{Br}} e^{s t} s^{\delta} d s \\
& =\frac{t^{-1-\delta}}{2 \pi i} \int_{\mathrm{Br}} e^{\sigma} \sigma^{\delta} d \sigma .
\end{aligned}
$$

The Bromwich contour $\mathrm{Br}$ can be deformed into the Hankel contour $\mathrm{Ha}$ [33], the latter winding around the negative real axis in the anticlockwise sense. In other words, Ha is a path starting at $\sigma=-\infty-i a$ ( $a>0$ and arbitrary) that encircles the branch cut laying on the negative real axis and ends up at $\sigma=-\infty+i b(b>0$ and arbitrary) [61]. Thus

$$
\mathcal{L}^{-1}\left\{s^{\delta}\right\}=\frac{t^{-1-\delta}}{2 \pi i} \int_{\mathrm{Ha}} e^{\sigma} \sigma^{\delta} d \sigma,
$$

and, by using the Hankel representation of the reciprocal of the Gamma function $[61,62]$

$$
\frac{1}{\Gamma(-\delta)}=\frac{1}{2 \pi i} \int_{\mathrm{Ha}} e^{\sigma} \sigma^{\delta} d \sigma,
$$

we readily obtain Eq. (A1)

We now expand the right-hand side of Eq. (56)

$$
\hat{p}(x, s) \simeq \frac{\sqrt{\lambda / 2}}{v} \sum_{n=0}^{\infty} \frac{(-1)^{n}}{n !}\left(\frac{|x| \sqrt{\lambda / 2}}{v}\right)^{n} s^{-1+(n+1) \alpha / 2} .
$$

Laplace inverting this expression and using Eq. (A1) we get

$$
\begin{aligned}
p(x, t) \simeq & \frac{\sqrt{\lambda / 2}}{v} \sum_{n=0}^{\infty} \frac{(-1)^{n}}{n !}\left(\frac{|x| \sqrt{\lambda / 2}}{v}\right)^{n} \\
& \times \frac{t^{-(n+1) \alpha / 2}}{\Gamma[1-(n+1) \alpha / 2]},
\end{aligned}
$$

which is an asymptotic approximation valid for large values of $t$ such that $t \gg \lambda^{-\alpha}$. Finally the definition of Mainardi function given in Eq. (59) directly leads to Eq. (58). 
[1] J. J. Duderstadt and W. R. Martin, Transport Theory (John Wiley, New York, 1979).

[2] G. H. Weiss, Physica A 311, 381 (2002).

[3] M. Shlesinger, J. Klafter, and G. Zumofen, Fractals 3, 491 (1995).

[4] J. B. Keller, Proc. Natl. Acad. Sci. USA 101, 1120 (2004).

[5] M. Abdel-Aziz and S. Gavin, Phys. Rev. C 70, 034905 (2004).

[6] J. Dunkel, P. Talkner, and P. Hänggi, Phys. Rev. D 75, 043001 (2007).

[7] J. Masoliver and G. H. Weiss, Eur. J. Phys. 17, 190 (1996).

[8] D. D. Joseph and L. Preziosi, Rev. Mod. Phys. 61, 41 (1989); 62, 375 (1990).

[9] D. Jou, J. Casas-Vázquez, and G. Lebon, Extended Irreversible Thermodynamics, 4th ed. (Springer, Berlin, 2010).

[10] V. A. Cimmelli, A. Sellitto, and D. Jou, Phys. Rev. B 81, 054301 (2010).

[11] V. Méndez, D. Campos, and W. Horsthemke, Phys. Rev. E 90, 042114 (2014).

[12] J. Fort and V. Méndez, Phys. Rev. Lett. 82, 867 (1999).

[13] J. Masoliver, K. Lindenberg, and G. H. Weiss, Physica A 157, 891 (1989).

[14] M. Kac, Mt. Rocky J. Math. 4, 497 (1974).

[15] G. H. Weiss, Aspects and Applications of the Random Walk (North-Holland, Amsterdam, 1994).

[16] A. Ishimaru, J. Opt. Soc. Am. 68, 1045 (1978); Appl. Opt. 28, 2210 (1989).

[17] S. I. Heizler, Nucl. Sci. Eng. 166, 17 (2010).

[18] S. Havlin and D. ben-Avraham, Adv. Phys. 36, 695 (1987).

[19] J. P. Bouchaud and A. Georges, Phys. Rep. 195, 127 (1990).

[20] R. Metzler and J. Klafter, Phys. Rep. 339, 1 (2000).

[21] B. J. West, M. Bologna, and P. Grigolini, Physics of Fractal Operators (Springer, Berlin, 2003).

[22] R. Metzler and J. Klafter, J. Phys. A 37, 161 (2004).

[23] R. Balescu, Aspects of Anomalous Transport in Plasmas (Taylor $\&$ Francis, London, 2005).

[24] I. I. Eliazar and M. F. Shlesinger, Phys. Rep. 527, 101 (2013).

[25] B. J. West, Rev. Mod. Phys. 86, 1169 (2014).

[26] J. Klafter and I. Sokolov, Physics World, Aug. 2005, p. 1.

[27] E. W. Montroll and G. H. Weiss, J. Math. Phys. 6, 167 (1965).

[28] H. Scher and E. W. Montroll, J. Stat. Phys. 9, 101 (1973).

[29] H. Scher and E. W. Montroll, Phys. Rev. B 12, 2455 (1975).

[30] D. ben-Avraham and S. Havlin, Diffusion and Reactions in Fractals and Disordered Systems (Cambridge University Press, Cambridge, 2000).

[31] R. Gorenflo and F. Mainardi, in Fractals and Fractional Calculus in Continuum Mechanics, edited by A. Carpinteri and F. Mainardi (Springer, Berlin, 1997), p. 1.

[32] I. Podbury, Fractional Differential Equations (Academic Press, San Diego, 1999).

[33] F. Mainardi, Appl. Math. Lett. 9, 23 (1996).

[34] F. Mainardi, Y. Luchko, and G. Pagnini, Fract. Calc. Appl. Anal. 4, 153 (2001).
[35] R. Gorenflo, F. Mainardi, and A. Vivoli, Chaos Solitons Fractals 34, 87 (2007).

[36] R. Balescu, Chaos Solitons Fractals 34, 62 (2007).

[37] R. C. Ball, S. Havlin, and G. H. Weiss, J. Phys. A 20, 4055 (1987).

[38] Because of Tauberian theorems (see Ref. [59]), large times and distances, $t \rightarrow \infty$ and $|\mathbf{r}| \rightarrow \infty$, correspond to small Laplace and Fourier variables, $s \rightarrow 0$ and $|\omega| \rightarrow 0$.

[39] A. Rebenshtok, S. Denisov, P. Hänggi, and E. Barkai, Phys. Rev. E 90, 062135 (2014).

[40] J. Masoliver, J. M. Porrà, and G. H. Weiss, Physica A 182, 593 (1992)

[41] J. M. Porrà, J. Masoliver, and G. H. Weiss, Physica A 218, 229 (1995).

[42] S. Godoy and L. S. García-Colín, Phys. Rev. E 55, 2127 (1997).

[43] M. Boguna, J. M. Porrà, and J. Masoliver, Phys. Rev. E 58, 6992 (1998).

[44] J. Masoliver, J. M. Porrà, and G. H. Weiss, Physica A 193, 469 (1993).

[45] A. Kolesnik and M. A. Pinsky, J. Stat. Phys. 142, 828 (2011).

[46] J. M. Porrà, J. Masoliver, and G. H. Weiss, Phys. Rev. E 55, 7771 (1997).

[47] E. Orshinger and X. Zhao, Chin. Ann. Math. 24, 1 (2003).

[48] E. Orshinger and L. Beghin, Probab. Theor. Relat. Fields 128, 141 (2004).

[49] M. D’Ovidio, E. Orshinger, and B. Toaldo, Stochastic Anal. Appl. 32, 1009 (2014).

[50] A. Compte and R. Metzler, J. Phys. A 30, 7277 (1997).

[51] R. Metzler and A. Compte, Physica A 268, 454 (1999).

[52] R. Metzler and T. F. Nonnenmacher, Phys. Rev. E 57, 6409 (1998).

[53] J. W. Hauss and K. W. Kehr, J. Phys. Chem. Solids 40, 1019 (1979).

[54] M. Shlesinger, Solid State Commun. 32, 1207 (1979).

[55] J. Masoliver and G. H. Weiss, Phys. Rev. E 49, 3852 (1994).

[56] J. F. C. Kingman, Poisson Processes (Oxford University Press, Oxford, 2002).

[57] G. E. Roberts and H. Kaufman, Table of Laplace Transforms (W. B. Saunders, Philadelphia, 1966).

[58] A. Erdelyi et al., Higher Transcendental Functions, Vol. 3 (McGraw-Hill, New York, 1953).

[59] R. A. Handelsman and J. S. Lew, SIAM J. Math. Anal. 5, 425 (1974).

[60] F. Mainardi and G. Pagnini, J. Comp. Appl. Math. 207, 245 (2007).

[61] T. Schmelzer and L. N. Trefethen, SIAM J. Numer. Anal. 45, 558 (2007).

[62] A. Erdelyi et al., Higher Transcendental Functions, Vol. 1 (McGraw-Hill, New York, 1953).

[63] R. Wong and Y.-Q. Zhao, Constr. Approx. 18, 355 (2002).

[64] R. B. Paris, Proc. R. Soc. London A 458, 3041 (2002). 